\title{
ASSOCIATION BETWEEN LABORATORY MARKERS AND PRESENCE OF CORONARY ARTERY DISEASE
}

\author{
Vladimir Kincla*, Roman Panovsky ${ }^{a}$, Jaroslav Meluzina, Jiri Semenka ${ }^{a}$, Ladislav Groch ${ }^{a}$, \\ Daniela Tomcikova $^{\mathrm{b}}$, Jiri Jarkovsky ${ }^{\mathrm{b}}$, Ladislav Dusek ${ }^{\mathrm{b}}$
}

\author{
a Ist Department of Internal Medicine-Cardioangiology, St. Ann Faculty Hospital, Masaryk University and International \\ Clinical Research Center, Pekarska 53, 65691 Brno, Czech Republic \\ ${ }^{b}$ Institute of Biostatistics and Analyses, Masaryk University, Kamenice 3, Brno \\ E-mail: vladimir.kincl@fnusa.cz
}

Received: March 19, 2010; Accepted: July 1, 2010

Key words: Coronary artery disease/Laboratory markers/Atherosclerosis

The aim of this paper is to elucidate the relation between laboratory markers and coronary artery disease (CAD).

Methods. The study involved 1254 consecutive patients with suspected or known CAD referred for coronary angiography. The blood samples including blood cell count, C-reactive protein, fibrinogen, uric acid, creatinine, and lipid spectrum were obtained after overnight fasting. One hundred and thirty-three patients were excluded due to incomplete records or inacceptable laboratory values. Differences among groups were tested with one-way ANOVA and Bonferroni post-hoc test for continuous variables and with chi-square test for categorical variables. Univariate and multivariate logistic regression was adopted for the analysis of risk factors and development of models for classification of patients into clinical categories.

Results. The linear logistic regression showed association of patient's biochemical markers with the presence of disease. Both acute and chronic CAD were associated with leukocyte count (Odds ratios 1.45 and 1.26), CRP (1.13; $1.05)$, fibrinogen $(4.23 ; 1.95)$, uric acid $(1.27 ; 1.38)$, creatinine (1.04; 1.04), HDL cholesterol (0.07; 0.12), triglycerides $(1.4 ; 1.52)$ and glucose $(1.56 ; 1.39)$. Presence of insignificant atherosclerosis was influenced only by fibrinogen (OR 1.73), creatinine (1.02), HDL cholesterol (0.5) and glucose level (1.23). There was no difference between one- and multivessel disease in laboratory values.

Conclusion. Leukocyte count, CRP level, triglycerides and uric acid are associated with the presence of both acute and chronic ischaemic heart disease, but not with number of stenosed vessels. In addition, glycemia, HDL cholesterol and namely fibrinogen and creatinine have relation to occurence of insignificant atherosclerosis.

\section{INTRODUCTION}

The coronary artery disease (CAD) is one of the most frequent causes of mortality and morbidity in developed countries ${ }^{1}$. Coronary artery disease is caused by progress of atherosclerotic plagues in coronary arteries. The common risk factors of CAD are hypertension, hyperlipoproteinaemia, smoking and diabetes mellitus and also determined factors as age and male sex. Atherosclerosis is a complex process and it is considered to have an inflammatory background, so the association between levels of various inflammatory markers and occurence, severity, and clinical form CAD have been studied ${ }^{2-6}$. There are many evidences that inflammation plays a key role in the patogenesis of stable $\mathrm{CAD}^{6-8}$ and acute coronary syndromes $^{9-12}$. The most frequently studied parameters were leukocyte count ${ }^{2,5,6,8,11,13-15}$, C-reactive protein (CRP) (ref. ${ }^{3,4,7,10}$ ), fibrinogen ${ }^{16,17}$, and also uric acid ${ }^{17-19}$. There were found association of high hs-CRP levels and increased risk of further coronary events ${ }^{7,10}$. Danesh et al. ${ }^{16}$ also found significant association of fibrinogen, CRP, albumin and leukocyte count with coronary artery disease by metaanalysis of prospective studies of CAD. Relation between increased level of uric acid and increased mortal- ity in CAD patients was also described by Bickel et al. ${ }^{19}$. The aim of this study was to evaluate the association of leukocyte count, high sensitivity CRP (hsCRP), fibrinogen, and other laboratory parameters like creatinine and uric acid in patients with acute coronary syndromes and stable CAD and with multi - and singlevessel disease in stable CAD subgroups.

\section{METHODS}

\section{Study population}

The study involved 1254 patients with suspected or known CAD referred for coronary angiography. The group of patients with CAD consisted of those with chronic stable CAD and acute coronary syndromes as well.

During the hospitalization the patients underwent full cardiologic investigation (history, physical examination, electrocardiography, laboratory examination, coronary angiography, echocardiography in patients with unclear diagnosis). All blood samples were taken after overnight fasting. The analysis of blood count was performed on analyzer SysMed XE 2100, Japan, fibrinogen level was determined according to Clause method on system BCS 
XP, Siemens, Germany. The values of CRP, creatinine, lipid spectrum, uric acid were determined on analyzer Advia 1650, Siemens, Germany, by using kits BLW and BioVendor. Patients with severe renal (creatinine over 200 umol/1) or hepatic failure, anemia, endocrine or neurological diseases or malignancies were excluded. We also excluded patients with smooth coronary arteries having the evidence of myocardial infarction or spastic angina pectoris or patients with uncomplete laboratory and other examinations results. The history of myocardial infarcion or unstable angina within one month was classified as acute coronary syndrome. The hyperlipoproteinemia was defined as known diagnosis of hyperlipoproteinemia in patient's documentation and/or hypolipidemic treatment or total cholesterol level above $5 \mathrm{mmol} / \mathrm{l}$ in blood samples, diabetes mellitus as current treatment with hypoglycemic drugs or diet or repeat fasting glucose $>7.0 \mathrm{mmol} / 1$ during hospitalization, hypertension as current treatment with antihypertensive drugs or repeat resting blood pressure $>140 / 90 \mathrm{mmHg}$ during hospitalization. All patients in-

Table 1a. Proportion of patients in groups according to CAD presence or absence and severity before and after data validation.

\begin{tabular}{|c|c|c|c|c|c|c|}
\hline \multirow{2}{*}{ Group } & \multirow{2}{*}{ Sub-group } & \multicolumn{2}{|c|}{ Before validation } & \multicolumn{2}{|c|}{ After validation } & \multirow{2}{*}{$\begin{array}{c}\text { No of } \\
\text { excluded } \\
\text { patients }\end{array}$} \\
\hline & & $\mathbf{N}$ & $\%$ & $\mathbf{N}$ & $\%$ & \\
\hline Acute CAD & & 270 & 21.5 & 249 & 22.2 & 21 \\
\hline \multirow[t]{3}{*}{ Chronic CAD } & & 642 & 51.2 & 568 & 50.7 & 74 \\
\hline & Singlevessel chronic CAD & 206 & 16.4 & 177 & 15.8 & 29 \\
\hline & Multivessel chronic CAD & 436 & 34.8 & 391 & 34.9 & 45 \\
\hline $\begin{array}{l}\text { Insignificant } \\
\text { atherosclerosis }\end{array}$ & & 126 & 10.0 & 109 & 9.7 & 17 \\
\hline Control group & & 216 & 17.2 & 195 & 17.4 & 21 \\
\hline Total & & 1254 & 100.0 & 1121 & 100.0 & 133 \\
\hline
\end{tabular}

CAD - coronary artery disease, insignificant atherosclerosis - stenosis $<50 \%$

Table 1b. Description of patients in study with comparison of acute and chronic CAD (basic characteristics and risk factors).

\begin{tabular}{|c|c|c|c|c|c|c|}
\hline & All patients & Acute CAD & Chronic CAD & $\begin{array}{c}\text { Insignificant } \\
\text { atherosclerosis }\end{array}$ & Control group \\
\hline & & $(\mathrm{N}=1121)$ & $(\mathrm{N}=249)$ & $(\mathrm{N}=568)$ & $(\mathrm{N}=109)$ & $(\mathrm{N}=195)$ \\
\hline \multirow{6}{*}{ 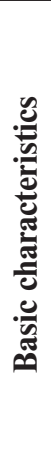 } & Male sex ${ }^{\dagger \dagger}$ & 70.0 & 70.7 & 77.5 & 64.2 & 50.8 \\
\hline & Age $(\mathrm{yrs})^{\dagger \dagger}$ & $\begin{array}{c}64.8 \\
(64.2 ; 65.4) \\
\end{array}$ & $\begin{array}{c}65.9 \\
(64.6 ; 67.2)^{*}\end{array}$ & $\begin{array}{c}65.7 \\
(64.9 ; 66.5)^{*}\end{array}$ & $\begin{array}{c}65.7 \\
(64 ; 67.3)^{*}\end{array}$ & $\begin{array}{c}60 \\
(58.6 ; 61.4)\end{array}$ \\
\hline & Height (cm) & $\begin{array}{c}171.3 \\
(170.8 ; 171.8)\end{array}$ & $\begin{array}{c}171.3 \\
(170.2 ; 172.4)\end{array}$ & $\begin{array}{c}171.9 \\
(171.2 ; 172.6)\end{array}$ & $\begin{array}{c}170.7 \\
(168.9 ; 172.5)\end{array}$ & $\begin{array}{c}170 \\
(168.7 ; 171.2)\end{array}$ \\
\hline & Weight (kg) & $\begin{array}{c}84.2 \\
(83.4 ; 85.1) \\
\end{array}$ & $\begin{array}{c}84.2 \\
(82.4 ; 86) \\
\end{array}$ & $\begin{array}{c}84.7 \\
(83.5 ; 85.9) \\
\end{array}$ & $\begin{array}{c}83.7 \\
(81 ; 86.3) \\
\end{array}$ & $\begin{array}{c}83.2 \\
(81.2 ; 85.3) \\
\end{array}$ \\
\hline & EF (\%) & $\begin{array}{c}53.4 \\
(52.6 ; 54.2)\end{array}$ & $\begin{array}{c}48.8 \\
(47.3 ; 50.4) * \S^{+}\end{array}$ & $\begin{array}{c}52.7 \\
(51.6 ; 53.8)^{*+}\end{array}$ & $\begin{array}{c}58.9 \\
(56.7 ; 61.1)\end{array}$ & $\begin{array}{c}58.2 \\
(56.5 ; 59.9)\end{array}$ \\
\hline & BMI $\left(\mathrm{kg} / \mathrm{m}^{2}\right)$ & $\begin{array}{c}28.7 \\
(28.4 ; 28.9)\end{array}$ & $\begin{array}{c}28.6 \\
(28.1 ; 29.2) \\
\end{array}$ & $\begin{array}{c}28.6 \\
(28.3 ; 28.9) \\
\end{array}$ & $\begin{array}{c}28.7 \\
(27.9 ; 29.5)\end{array}$ & $\begin{array}{c}28.8 \\
(28.1 ; 29.4)\end{array}$ \\
\hline \multirow{6}{*}{ 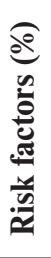 } & Hypertension ${ }^{\dagger}$ & 78.1 & 79.1 & 79.8 & 81.7 & 69.7 \\
\hline & Diabetes mellitus $^{\dagger \dagger}$ & 28.4 & 34.5 & 31.5 & 22.9 & 14.4 \\
\hline & Hyperlipoproteinemia $^{\dagger \dagger}$ & 78.5 & 79.9 & 84.3 & 67.0 & 66.2 \\
\hline & Perifery artery disease $e^{\dagger \dagger}$ & 8.1 & 15.3 & 8.6 & 0.9 & 1.5 \\
\hline & History of stroke & 9.6 & 14.1 & 10.7 & 5.5 & 3.1 \\
\hline & Smoking experience ${ }^{\dagger \dagger}$ & 47.1 & 52.6 & 50.7 & 36.7 & 35.4 \\
\hline
\end{tabular}

cathegorical variables are presented in $\%$, continuous variables as mean ( $95 \%$ confidence interval)

$\dagger$ significant difference among groups - ANOV A / Chi-square test $(p<0.05)$,

${ }^{\dagger \dagger}$ significant difference among groups - ANOVA / Chi-square test $(\mathrm{p}<0.001)$,

* significant difference vs. control group - Bonferroni test $(\mathrm{p}<0.001)$

$\S$ significant difference vs. chronic CAD - Bonferroni test $(p<0.001)$

+ significant difference vs. insignificant atherosclerosis - Bonferroni test $(p<0.001)$

EF - left ventricular ejection fraction, BMI - body mass index 
volved in this study gave informed consent and study was approved by institutional ethics comittee.

\section{Coronary angiography}

The coronary angiography was performed by femoral or radial artery approach using angiographic device Philips Allura Xper FD 10 (Philips, The Netherlands). The examinations were evaluated by two experienced cardiologists. Coronary artery disease was defined as more than $50 \%$ luminar diameter stenosis of at least one coronary artery. Patients with CAD were divided into groups of chronic stable CAD and acute coronary syndromes as described above, patients in stable CAD group were divided into subgroups with single- or multivessel disease.

Patients with insignificant atherosclerosis (luminal diameter narrowing below 50\%) were given into separate group. Patients with smooth coronary arteries were taken as controls.

\section{Data validation and statistical analysis:}

One thousand two hundred and fifty-four patients were included into the study. After data evaluation 133 patients were excluded due to incomplete records or inacceptable laboratory values. In data evaluation crucial parameters were age, gender, body mass index (BMI), data from patient's history (history of hypertension, diabetes mellitus, stroke, known hyperlipoproteinemia, perifery artery disease, renal insufficiency and smoking) and biochemical parameters (hemoglobin, leucocytes, trombocytes, fibrinogen, prothrombin test, total cholesterol, low density lipoprotein (LDL) cholesterol, high density lipoproteid (HDL) cholesterol, triglycerides, uric acid, creatinine, glycemia, CRP). Finally, patients without performed examination from ventriculography were excluded due to not accessible value of left ventricular ejection fraction. Number of patients in the groups are presented in Table 1a.

Table 2. Laboratory markers in groups of acute and chronic CAD.

\begin{tabular}{|c|c|c|c|c|c|c|}
\hline & All patients & Acute CAD & Chronic CAD & $\begin{array}{l}\text { Insignificant } \\
\text { atherosclerosis }\end{array}$ & Control group \\
\hline & & $(\mathrm{N}=1121)$ & $(\mathrm{N}=249)$ & $(\mathrm{N}=568)$ & $(\mathrm{N}=109)$ & $(\mathrm{N}=195)$ \\
\hline \multirow{12}{*}{ 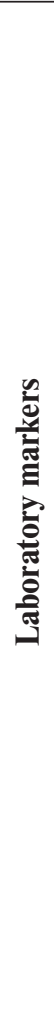 } & $\begin{array}{l}\text { Leukocytes }^{\mathrm{a} \dagger \dagger} \\
{\left[\times 10^{9} / 1\right]}\end{array}$ & $\begin{array}{c}7.5 \\
(7.3 ; 7.6) \\
\end{array}$ & $\begin{array}{c}8.2 \\
(7.9 ; 8.5) \S++* *\end{array}$ & $\begin{array}{c}7.5 \\
(7.3 ; 7.6)^{* *}\end{array}$ & $\begin{array}{c}7 \\
(6.7 ; 7.3) \\
\end{array}$ & $\begin{array}{c}6.7 \\
(6.5 ; 7)\end{array}$ \\
\hline & $\begin{array}{l}\text { C-reactive protein }{ }^{\mathrm{a} \dagger \dagger} \\
{[\mathrm{mg} / \mathrm{l}]}\end{array}$ & $\begin{array}{c}4.3 \\
(4 ; 4.6)\end{array}$ & $\begin{array}{c}7.9 \\
(6.8 ; 9.3) \S++* *\end{array}$ & $\begin{array}{c}3.8 \\
(3.5 ; 4.1)^{*}\end{array}$ & $\begin{array}{c}3.5 \\
(2.9 ; 4.2)\end{array}$ & $\begin{array}{c}2.8 \\
(2.5 ; 3.2)\end{array}$ \\
\hline & $\begin{array}{l}\text { Fibrinogen }^{\dagger \dagger} \\
{[\mathrm{g} / \mathrm{l}]}\end{array}$ & $\begin{array}{c}4 \\
(4 ; 4.1)\end{array}$ & $\begin{array}{c}4.4 \\
(4.3 ; 4.5) \S++* *\end{array}$ & $\begin{array}{c}4 \\
(3.9 ; 4)^{* *}\end{array}$ & $\begin{array}{c}3.9 \\
(3.8 ; 4) *\end{array}$ & $\begin{array}{c}3.7 \\
(3.6 ; 3.8)\end{array}$ \\
\hline & $\begin{array}{l}\text { Uric acid }^{\mathrm{a} \dagger} \\
{[\text { umol/l] }}\end{array}$ & $\begin{array}{c}355.2 \\
(349.6 ; 361)\end{array}$ & $\begin{array}{c}356.2 \\
(343.5 ; 369.3)\end{array}$ & $\begin{array}{c}362.5 \\
(354.7 ; 370.6)^{*}\end{array}$ & $\begin{array}{c}352.7 \\
(336.7 ; 369.4)\end{array}$ & $\begin{array}{c}335.1 \\
(322.4 ; 348.3)\end{array}$ \\
\hline & $\begin{array}{l}\text { Creatinine }^{\mathrm{a}^{\dagger} \dagger} \\
\text { [umol/l] }\end{array}$ & $\begin{array}{c}100.3 \\
(99.3 ; 101.2) \\
\end{array}$ & $\begin{array}{c}102.4 \\
(100.3 ; 104.5)^{* *}\end{array}$ & $\begin{array}{c}102.1 \\
(100.7 ; 103.5)^{* *}\end{array}$ & $\begin{array}{c}98.7 \\
(95.9 ; 101.7)^{*}\end{array}$ & $\begin{array}{c}93.3 \\
(91.3 ; 95.3) \\
\end{array}$ \\
\hline & $\begin{array}{l}\text { Cholesterol }^{\dagger} \\
{[\mathrm{mmol} / \mathrm{l}]}\end{array}$ & $\begin{array}{c}4.5 \\
(4.4 ; 4.6) \\
\end{array}$ & $\begin{array}{c}4.4 \\
(4.3 ; 4.6)^{*} \\
\end{array}$ & $\begin{array}{c}4.4 \\
(4.4 ; 4.5)^{*} \\
\end{array}$ & $\begin{array}{c}4.6 \\
(4.4 ; 4.8) \\
\end{array}$ & $\begin{array}{c}4.7 \\
(4.6 ; 4.8) \\
\end{array}$ \\
\hline & $\begin{array}{l}\mathbf{L D L}^{\mathrm{a}} \\
{[\mathrm{mmol} / \mathrm{l}]}\end{array}$ & $\begin{array}{c}2.5 \\
(2.4 ; 2.5) \\
\end{array}$ & $\begin{array}{c}2.5 \\
(2.4 ; 2.6) \\
\end{array}$ & $\begin{array}{c}2.4 \\
(2.4 ; 2.5)^{*} \\
\end{array}$ & $\begin{array}{c}2.5 \\
(2.4 ; 2.7) \\
\end{array}$ & $\begin{array}{c}2.6 \\
(2.5 ; 2.7) \\
\end{array}$ \\
\hline & $\begin{array}{l}\mathrm{HDL}^{\mathrm{a} \dagger \dagger} \\
{[\mathrm{mmol} / \mathrm{l}]}\end{array}$ & $\begin{array}{c}1.1 \\
(1.1 ; 1.2)\end{array}$ & $\begin{array}{c}1.1 \\
(1 ; 1.1)++* *\end{array}$ & $\begin{array}{c}1.1 \\
(1.1 ; 1.1)++* *\end{array}$ & $\begin{array}{c}1.2 \\
(1.2 ; 1.3)\end{array}$ & $\begin{array}{c}1.3 \\
(1.3 ; 1.4)\end{array}$ \\
\hline & $\begin{array}{l}\text { Triglycerides }^{\mathrm{a}^{\dagger}} \\
{[\mathrm{mmol} / \mathrm{l}]}\end{array}$ & $\begin{array}{c}1.5 \\
(1.5 ; 1.6) \\
\end{array}$ & $\begin{array}{c}1.6 \\
(1.5 ; 1.6)^{*}\end{array}$ & $\begin{array}{c}1.6 \\
(1.5 ; 1.7)^{*}\end{array}$ & $\begin{array}{c}1.5 \\
(1.3 ; 1.6) \\
\end{array}$ & $\begin{array}{c}1.4 \\
(1.3 ; 1.5)\end{array}$ \\
\hline & $\begin{array}{l}\text { Glucose }^{\mathrm{a} \dagger \dagger} \\
{[\mathrm{mmol} / \mathrm{l}]}\end{array}$ & $\begin{array}{c}5.7 \\
(5.6 ; 5.8) \\
\end{array}$ & $\begin{array}{c}6.1 \\
(5.9 ; 6.4) \S+* *\end{array}$ & $\begin{array}{c}5.7 \\
(5.6 ; 5.8)^{* *}\end{array}$ & $\begin{array}{c}5.5 \\
(5.2 ; 5.7) \\
\end{array}$ & $\begin{array}{c}5.2 \\
(5 ; 5.3) \\
\end{array}$ \\
\hline & $\begin{array}{l}\text { Trombocytes }^{\mathrm{a}^{\mathrm{\dagger}}} \\
{[\mathrm{x}} \\
\end{array}$ & $\begin{array}{c}202.1 \\
(198.7 ; 205.4) \\
\end{array}$ & $\begin{array}{c}214.7 \\
(206.6 ; 223) \S \\
\end{array}$ & $\begin{array}{c}196.4 \\
(191.9 ; 201) \\
\end{array}$ & $\begin{array}{c}199.5 \\
(189 ; 210.5) \\
\end{array}$ & $\begin{array}{c}204.7 \\
(197.7 ; 211.9) \\
\end{array}$ \\
\hline & $\begin{array}{l}\text { Hemoglobin } \\
{[\mathrm{g} / \mathrm{l}]}\end{array}$ & $\begin{array}{c}138.1 \\
(137.2 ; 138.9)\end{array}$ & $\begin{array}{c}135.1 \\
(133.1 ; 137) \S\end{array}$ & $\begin{array}{c}139.5 \\
(138.3 ; 140.7)\end{array}$ & $\begin{array}{c}138.5 \\
(136.3 ; 140.7)\end{array}$ & $\begin{array}{c}137.5 \\
(135.6 ; 139.4)\end{array}$ \\
\hline
\end{tabular}

cathegorical variables are presented in \%, continuous variables as mean ( $95 \%$ confidence interval)

a due to not normal distribution logaritmic transformation of the data was needed,

$\dagger$ significant difference among groups - ANOVA / Chi-square test $(p<0.050)$,

${ }^{\dagger \dagger}$ significant difference among groups - ANOVA / Chi-square test $(p<0.001)$,

${ }^{\S}$ significant difference between acute CAD and chronic CAD - Bonferroni test $(\mathrm{p}<0.001)$,

${ }^{+}$significant difference vs. insignificant atherosclerosis - Bonferroni test $(p<0.05)$,

${ }^{++}$significant difference vs. insignificant atherosclerosis - Bonferroni test $(p<0.001)$,

* significant difference vs. control group - Bonferroni test $(p<0.05)$

$* *$ significant difference vs. control group - Bonferroni test $(p<0.001)$ 
Basic statistical description of study population adopted percentage proportions for categorical parameters while the continuous variables were expressed as mean and $95 \%$ confidence interval or median and percentile range. Significant differences among groups were tested with one-way ANOVA and Bonferroni post-hoc test for continuous variables and with chi-square test for categorical variables.

Bonferonni post-hoc test was adopted as a solution for multiple testing problem; it is the correction for increased level of type I error during multiple testing with objective to reach $=0.05$ for all tests. Predictors in logistic regression were described by their odds ratio and confidence interval; their statistical significance was tested using Wald test which is a standard test for testing whether an independent variable has a statistically significant relationship with a dependent variable. Statistical significance of the whole logistic models was tested by means of Hosmer and Lemeshow test; the null hypothesis of the test is that there is no difference between the observed and predicted values of the dependent variable. If its $p>0.05$ we fail to reject the null hypothesis that there is no difference, implying that the model's estimates fit the data at an acceptable level. Analyses were performed using SPSS 12.0 and Statistica 8.0.

\section{RESULTS}

Proportion of patients in groups according to CAD presence or absence and severity before and after data validation shows Table 1a. Patients with both acute and

Table 3. Laboratory markers in subgroups of multivessel and singlevessel CAD.

\begin{tabular}{|c|c|c|c|c|c|}
\hline & All patients & $\begin{array}{l}\text { Singlevessel chronic } \\
\text { CAD }\end{array}$ & $\begin{array}{l}\text { Multivessel chronic } \\
\text { CAD }\end{array}$ & Control group \\
\hline & & $(\mathrm{N}=763)$ & $(\mathrm{N}=177)$ & $(\mathrm{N}=391)$ & $(\mathrm{N}=195)$ \\
\hline \multirow{12}{*}{ 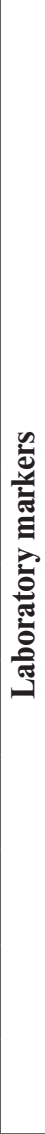 } & $\begin{array}{l}\text { Leukocytes }^{\mathrm{a} \dagger \dagger} \\
{\left[\begin{array}{ll}\mathbf{x} & \left.10^{\circ} / \mathrm{I}\right]\end{array}\right.}\end{array}$ & $\begin{array}{c}7.3 \\
(7.2 ; 7.4)\end{array}$ & $\begin{array}{c}7.6 \\
(7.3 ; 7.9) \S \S\end{array}$ & $\begin{array}{c}7.4 \\
(7.2 ; 7.6) \S \S\end{array}$ & $\begin{array}{c}6.7 \\
(6.5 ; 7)\end{array}$ \\
\hline & $\begin{array}{l}\text { C-reactive protein }{ }^{\mathrm{a} \dagger} \\
{[\mathrm{mg} / \mathrm{l}]}\end{array}$ & $\begin{array}{c}3.5 \\
(3.3 ; 3.8)\end{array}$ & $\begin{array}{c}3.8 \\
(3.3 ; 4.5) \S\end{array}$ & $\begin{array}{c}3.8 \\
(3.4 ; 4.2) \S\end{array}$ & $\begin{array}{c}2.8 \\
(2.5 ; 3.2)\end{array}$ \\
\hline & $\begin{array}{l}\text { fibrinogen }^{\dagger \dagger} \\
{[\mathrm{g} / \mathrm{l}]}\end{array}$ & $\begin{array}{c}3.9 \\
(3.9 ; 3.9) \\
\end{array}$ & $\begin{array}{c}3.9 \\
(3.8 ; 4) \S \\
\end{array}$ & $\begin{array}{c}4 \\
(3.9 ; 4.1) \S \S \\
\end{array}$ & $\begin{array}{c}3.7 \\
(3.6 ; 3.8) \\
\end{array}$ \\
\hline & $\begin{array}{l}\text { uric acid }^{a \dagger} \\
\text { [umol/l] }\end{array}$ & $\begin{array}{c}355.3 \\
(348.5 ; 362.2) \\
\end{array}$ & $\begin{array}{c}369 \\
(353.9 ; 384.7) \S \\
\end{array}$ & $\begin{array}{c}359.6 \\
(350.5 ; 369) \S \\
\end{array}$ & $\begin{array}{c}335.1 \\
(322.4 ; 348.3)\end{array}$ \\
\hline & $\begin{array}{l}\text { Creatinine }^{\mathrm{a} \dagger \dagger} \\
{[\text { umol/l] }}\end{array}$ & $\begin{array}{c}99.8 \\
(98.6 ; 101)\end{array}$ & $\begin{array}{c}100.9 \\
(98.6 ; 103.2) \S \S \\
\end{array}$ & $\begin{array}{c}102.7 \\
(101 ; 104.5) \S \S \\
\end{array}$ & $\begin{array}{c}93.3 \\
(91.3 ; 95.3) \\
\end{array}$ \\
\hline & $\begin{array}{l}\text { Total cholesterol } \\
{[\mathrm{mmol} / \mathrm{l}]}\end{array}$ & $\begin{array}{c}4.5 \\
(4.4 ; 4.6) \\
\end{array}$ & $\begin{array}{c}4.5 \\
(4.3 ; 4.6) \\
\end{array}$ & $\begin{array}{c}4.4 \\
(4.3 ; 4.5) \S \\
\end{array}$ & $\begin{array}{c}4.7 \\
(4.6 ; 4.8)\end{array}$ \\
\hline & $\begin{array}{l}\mathbf{L D L}^{\mathrm{a}} \\
{[\mathrm{mmol} / \mathrm{I}]}\end{array}$ & $\begin{array}{c}2.5 \\
(2.4 ; 2.5) \\
\end{array}$ & $\begin{array}{c}2.5 \\
(2.3 ; 2.6) \\
\end{array}$ & $\begin{array}{c}2.4 \\
(2.3 ; 2.5) \\
\end{array}$ & $\begin{array}{c}2.6 \\
(2.5 ; 2.7) \\
\end{array}$ \\
\hline & $\begin{array}{l}\mathrm{HDL}^{\mathrm{a} \dagger \dagger} \\
{[\mathrm{mmol} / \mathrm{l}]}\end{array}$ & $\begin{array}{c}1.2 \\
(1.1 ; 1.2)\end{array}$ & $\begin{array}{c}1.2 \\
(1.1 ; 1.2) \S \S\end{array}$ & $\begin{array}{c}1.1 \\
(1.1 ; 1.1) \S \S\end{array}$ & $\begin{array}{c}1.3 \\
(1.3 ; 1.4)\end{array}$ \\
\hline & $\begin{array}{l}\text { Triglycerides }^{\text {ał } \dagger} \\
{[\mathrm{mmol} / \mathrm{l}]}\end{array}$ & $\begin{array}{c}1.6 \\
(1.5 ; 1.6) \\
\end{array}$ & $\begin{array}{c}1.5 \\
(1.5 ; 1.6) \\
\end{array}$ & $\begin{array}{c}1.6 \\
(1.6 ; 1.7) \S \S \\
\end{array}$ & $\begin{array}{c}1.4 \\
(1.3 ; 1.5) \\
\end{array}$ \\
\hline & $\begin{array}{l}\text { Glucose }^{\mathrm{a} \dagger \dagger} \\
{[\mathrm{mmol} / \mathrm{l}]}\end{array}$ & $\begin{array}{c}5.6 \\
(5.5 ; 5.6) \\
\end{array}$ & $\begin{array}{c}5.5 \\
(5.4 ; 5.7) \S \\
\end{array}$ & $\begin{array}{c}5.8 \\
(5.6 ; 5.9) \S \S \\
\end{array}$ & $\begin{array}{c}5.2 \\
(5 ; 5.3) \\
\end{array}$ \\
\hline & $\begin{array}{l}\text { Trombocytes }^{\mathrm{a} \dagger} \\
{\left[\begin{array}{ll}\mathbf{x} & 10\end{array} / \mathrm{I}\right]} \\
\end{array}$ & $\begin{array}{c}198.5 \\
(194.7 ; 202.4) \\
\end{array}$ & $\begin{array}{c}203.8 \\
(196.5 ; 211.4) \\
\end{array}$ & $\begin{array}{c}193.1 \\
(187.6 ; 198.8) \S \\
\end{array}$ & $\begin{array}{c}204.7 \\
(197.7 ; 211.9) \\
\end{array}$ \\
\hline & $\begin{array}{l}\text { Hemoglobin }^{\dagger} \\
{[\mathrm{g} / \mathrm{l}]}\end{array}$ & $\begin{array}{c}139 \\
(138 ; 140)\end{array}$ & $\begin{array}{c}141.8 \\
(140 ; 143.6) * \S\end{array}$ & $\begin{array}{c}138.5 \\
(137 ; 139.9)^{*}\end{array}$ & $\begin{array}{c}137.5 \\
(135.6 ; 139.4)\end{array}$ \\
\hline
\end{tabular}

cathegorical variables are presented in \%, continuous variables as mean ( $95 \%$ confidence interval)

a due to not normal distribution logaritmic transformation of the data was needed,

$\dagger^{\dagger}$ significant difference among groups - ANOVA / Chi-square test $(p<0.050)$,

$\dagger$ significant difference among groups - ANOVA / Chi-square test $(p<0.001)$,

* significant difference between singlevessel and multivessel chronic CAD - Bonferroni test $(\mathrm{p}<0.05)$,

${ }^{\S}$ significant difference vs. control group - Bonferroni test $(\mathrm{p}<0.05)$

${ }_{\S}^{\S}$ significant difference vs. control group - Bonferroni test $(p<0.001)$ 
chronic CAD had significantly higher occurency of hypertension, diabetes, hyperlipoproteinemia, perifery artery disease, stroke, smoking experience and were markedly older. The left ventricular ejection fraction was also significantly lower in CAD patients (Table 1b).

The basic differences in laboratory parameters are presented in Table 2. Patients with acute forms of CAD had significantly higher leukocyte count, C-reactive protein and fibrinogen and also glucose. It is not surprising because inflammatory reaction is physiological in case of myocardial necrosis or atherosclerotic plaque rupture. Otherwise, in this parameters are also significant differences between patients with chronic CAD and controls that demonstrates the inflammatory background of atherosclerosis progress and development. From other parameters, patients with chronic CAD had higher level of uric acid, creatinine and lower HDL cholesterol. Results of lipid spectrum may seem paradoxical, because patients with CAD had lower total and LDL cholesterol, but this is caused by present hypolipidemic medication that was significantly more frequent in this group. The prevalence of hypolipidemic therapy was as follows: in chronic CAD group had 548 (96.5\%) patients hypolipidemic drugs (530 had statin, 1 fibrate, 17 combination statin+fibrate), in acute CAD group was on therapy 243 patients (97.6\%, 239 statin, 1 fibrate, 3 combination), in insignificant atherosclerosis group 83 patients $(76.1 \%$, all 83 had statin) and in control group had hypolipidemic drugs 49 patients $(25.2 \%$, 47 statin, 1 fibrate, 1 combination). Significant differences were found between groups with insignificant atherosclerosis and controls in fibrinogen and creatinine level only.

Detail division in subgroups with single- and multivessel chronic CAD is presented in Table 3. Inflammatory markers, uric acid and glucose were again significantly elevated both in one- and multivessel disease group against controls and HDL cholesterol was lower in this groups too. There was no significant difference between singleand multi vessel group in neither inflammatory markers nor other biochemical parameters, except for hemoglobin. This difference was probably casual, because patients with singlevessel CAD had higher hemoglobin level than those with multi-vessel but control group had lower than both groups with CAD.

The linear logistic regression showed association of patient's biochemical markers on presence of CAD (Table 4 ). Both acute and chronic CAD were associated with leukocyte count, CRP, fibrinogen, uric acid, creatinine, HDL cholesterol, triglycerides and glucose. On the other

Table 4. Influence of patient's biochemical parameters on presence of disease based on linear logistic regression.

\begin{tabular}{|c|c|c|c|c|c|c|}
\hline & $\begin{array}{l}\text { Acute } \\
\text { CAD }\end{array}$ & $\begin{array}{l}\text { Chronic } \\
\text { CAD }\end{array}$ & $\begin{array}{c}\text { Insignificant } \\
\text { atherosclerosis }\end{array}$ & $\begin{array}{l}\text { Singlevessel } \\
\text { chronic CAD }\end{array}$ & $\begin{array}{c}\text { Multivessel chronic } \\
\text { CAD }\end{array}$ \\
\hline & & $(\mathrm{N}=249)$ & $(\mathrm{N}=568)$ & $(N=109)$ & $(\mathrm{N}=177)$ & $(N=391)$ \\
\hline \multirow{12}{*}{ 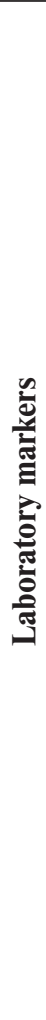 } & Leucocytes & $\begin{array}{c}1.45 \\
(1.30 ; 1.61)^{\dagger \dagger}\end{array}$ & $\begin{array}{c}1.26 \\
(1.14 ; 1.39)^{\dagger \dagger}\end{array}$ & $\begin{array}{c}1.08 \\
(0.95 ; 1.24) \\
\end{array}$ & $\begin{array}{c}1.29 \\
(1.15 ; 1.45)^{\dagger \dagger}\end{array}$ & $\begin{array}{c}1.24 \\
(1.12 ; 1.38)^{\dagger \dagger}\end{array}$ \\
\hline & C-reactive protein & $\begin{array}{c}1.13 \\
(1.08 ; 1.17)^{\dagger \dagger}\end{array}$ & $\begin{array}{c}1.05 \\
(1.01 ; 1.08)^{\dagger}\end{array}$ & $\begin{array}{c}1.03 \\
(0.99 ; 1.07)\end{array}$ & $\begin{array}{c}1.04 \\
(1.00 ; 1.07)^{\dagger}\end{array}$ & $\begin{array}{c}1.05 \\
(1.01 ; 1.09)^{\dagger}\end{array}$ \\
\hline & Fibrinogen & $\begin{array}{c}4.23 \\
(3.06 ; 5.86)^{\dagger \dagger}\end{array}$ & $\begin{array}{c}1.95 \\
(1.50 ; 2.54)^{\dagger \dagger}\end{array}$ & $\begin{array}{c}1.73 \\
(1.2 ; 2.51)^{\dagger}\end{array}$ & $\begin{array}{c}1.65 \\
(1.21 ; 2.26)^{\dagger}\end{array}$ & $\begin{array}{c}2.16 \\
(1.62 ; 2.88)^{\dagger \dagger}\end{array}$ \\
\hline & $\begin{array}{l}\text { Uric acid } \\
\text { (100 units) }\end{array}$ & $\begin{array}{c}1.27 \\
(1.05 ; 1.54)^{\dagger}\end{array}$ & $\begin{array}{c}1.38 \\
(1.15 ; 1.65)^{\dagger}\end{array}$ & $\begin{array}{c}1.21 \\
(0.94 ; 1.56)\end{array}$ & $\begin{array}{c}1.46 \\
(1.18 ; 1.82)^{\dagger \dagger}\end{array}$ & $\begin{array}{c}1.34 \\
(1.1 ; 1.62)^{\dagger}\end{array}$ \\
\hline & Creatinine & $\begin{array}{c}1.04 \\
(1.02 ; 1.05)^{\dagger \dagger}\end{array}$ & $\begin{array}{c}1.04 \\
(1.03 ; 1.05)^{\dagger \dagger}\end{array}$ & $\begin{array}{c}1.02 \\
(1.01 ; 1.04)^{\dagger} \\
\end{array}$ & $\begin{array}{c}1.03 \\
(1.02 ; 1.05)^{\dagger \dagger}\end{array}$ & $\begin{array}{c}1.04 \\
(1.03 ; 1.05)^{\dagger \dagger}\end{array}$ \\
\hline & Total cholesterol & $\begin{array}{c}0.79 \\
(0.66 ; 0.95)^{\dagger} \\
\end{array}$ & $\begin{array}{c}0.78 \\
(0.67 ; 0.91)^{\dagger} \\
\end{array}$ & $\begin{array}{c}0.88 \\
(0.69 ; 1.12) \\
\end{array}$ & $\begin{array}{c}0.78 \\
(0.64 ; 0.97)^{\dagger} \\
\end{array}$ & $\begin{array}{c}0.77 \\
(0.65 ; 0.91)^{\dagger} \\
\end{array}$ \\
\hline & LDL & $\begin{array}{c}0.89 \\
(0.72 ; 1.09) \\
\end{array}$ & $\begin{array}{c}0.79 \\
(0.66 ; 0.96)^{\dagger} \\
\end{array}$ & $\begin{array}{c}0.89 \\
(0.66 ; 1.2) \\
\end{array}$ & $\begin{array}{c}0.78 \\
(0.60 ; 1.02)\end{array}$ & $\begin{array}{c}0.79 \\
(0.65 ; 0.96)^{\dagger}\end{array}$ \\
\hline & HDL & $\begin{array}{c}0.07 \\
(0.04 ; 0.15)^{\dagger \dagger}\end{array}$ & $\begin{array}{c}0.12 \\
(0.07 ; 0.20)^{\dagger \dagger}\end{array}$ & $\begin{array}{c}0.50 \\
(0.26 ; 0.98)^{\dagger}\end{array}$ & $\begin{array}{c}0.23 \\
(0.12 ; 0.43)^{\dagger \dagger}\end{array}$ & $\begin{array}{c}0.08 \\
(0.04 ; 0.15)^{\dagger \dagger}\end{array}$ \\
\hline & Triglycerides & $\begin{array}{c}1.4 \\
(1.06 ; 1.84)^{\dagger}\end{array}$ & $\begin{array}{c}1.52 \\
(1.19 ; 1.94)^{\dagger \dagger}\end{array}$ & $\begin{array}{c}1.09 \\
(0.78 ; 1.53)\end{array}$ & $\begin{array}{c}1.33 \\
(0.99 ; 1.79)\end{array}$ & $\begin{array}{c}1.57 \\
(1.22 ; 2.02)^{\dagger \dagger}\end{array}$ \\
\hline & Glucose & $\begin{array}{c}1.56 \\
(1.33 ; 1.82)^{\dagger \dagger}\end{array}$ & $\begin{array}{c}1.39 \\
(1.20 ; 1.61)^{\dagger \dagger}\end{array}$ & $\begin{array}{c}1.23 \\
(1.02 ; 1.48)^{\dagger}\end{array}$ & $\begin{array}{c}1.30 \\
(1.09 ; 1.55)^{\dagger}\end{array}$ & $\begin{array}{c}1.42 \\
(1.22 ; 1.65)^{\dagger \dagger}\end{array}$ \\
\hline & $\begin{array}{l}\text { Trombocytes } \\
\text { (50 units) }\end{array}$ & $\begin{array}{c}1.12 \\
(1.02 ; 1.38)^{\dagger}\end{array}$ & $\begin{array}{c}0.9 \\
(0.79 ; 1.04)\end{array}$ & $\begin{array}{c}0.95 \\
(0.77 ; 1.17) \\
\end{array}$ & $\begin{array}{c}0.98 \\
(0.81 ; 1.19)\end{array}$ & $\begin{array}{c}0.87 \\
(0.76 ; 1.01)\end{array}$ \\
\hline & Hemoglobin & $\begin{array}{c}0.99 \\
(0.98 ; 1.00)\end{array}$ & $\begin{array}{c}1.01 \\
(1.00 ; 1.02)\end{array}$ & $\begin{array}{c}1.01 \\
(0.99 ; 1.02)\end{array}$ & $\begin{array}{c}1.03 \\
(1.01 ; 1.04)^{\dagger}\end{array}$ & $\begin{array}{c}1.00 \\
(0.99 ; 1.02)\end{array}$ \\
\hline
\end{tabular}

\footnotetext{
$\dagger$ Wald test, significance $\mathrm{p}<0.050$

$\doteqdot$ Wald test, significance $p<0.001$

Data are presented as odds ratios with $95 \%$ confidence interval
} 
hand, presence of insignificant atherosclerosis was influenced only by fibrinogen, creatinine, HDL cholesterol and glucose level. From clinical factors, significant relationship was found between age, male sex, hypertension, diabetes, hyperlipidemia, history of stroke, perifery artery disease, smoking experience and CAD (acute and chronic, singlevessel and multivessel as well). Insignificant atherosclerosis was associated only with male sex, age and hypertension.

\section{DISCUSSION}

In our study we tested several laboratory parameters and their association with the presence and severity of coronary atherosclerosis. We proved that elevated inflammatory markers as leukocyte count, C- reactive protein and fibrinogen are associated primarily with acute forms of coronary artery disease and presence of chronic CAD, but not with the number of affected vessels in chronic CAD. Patients with multivessel chronic CAD had rather lower mean leukocyte count that those with single vessel disease. Also other parameters (uric acid, creatinine, blood lipids and glucose) failed to distinguish the number of diseased coronary arteries. All mentioned parameters were significantly different between CAD and control group, only fibrinogen and creatinine also between insignificant atherosclerosis and control group. So we cannot confirm findings from previous studies, e.g. Cavusoglu et al. ${ }^{2}$, Sabatine et al. ${ }^{5}$, that described the association between leukocyte count and the number of significantly narrowed coronary arteries. The linkage between insignificant atherosclerosis and fibrinogen and creatinine level corresponded to previous findings. Levenson et al. ${ }^{20,21}$ demonstrated that prevalece of atherosclerotic plaques was significantly more pronounced with increasing tertile of fibrinogen levels. The authors also found the synergic effect between fibrinogen and total/HDL cholesterol ratio in formation of subclinical coronary and extracoronary atherosclerosis. The role of renal function and creatinine was described by several authors. Bartnicky et al. ${ }^{22}$ found out the increased odds ratio of coronary artery disease in patients with decreased glomerulal filtration. Cerne et al. ${ }^{23}$ described the mildly elevated serum creatinine to be associated with the extent of coronary atherosclerosis independently of conventional risk factors. Mild renal insuficiency is also associated with reduced coronary flow in patients with non-obstructive coronary artery disease. That can be caused by synchronous changes in the renal and coronary microcirculation ${ }^{24}$.

Our study has several limitations. The number of subjects was relatively large, but it was selected population referred for coronary angiography. Also influence of blood lipid spectrum could not be exactly examined due to the present hypolipidemic medication in CAD patients.

In conclusion, laboratory parameters as leukocyte count, CRP level, triglycerides and uric acid are associated with the presence of coronary artery disease (both acute and chronic) but not with the number of diseased vessels. In addition, glycemia, HDL cholesterol and namely fibrinogen and creatinine have relation to the occurence of insignificant atherosclerosis.

\section{ACKNOWLEDGEMENT}

Supported in part by a grant of Ministry of Education of the Czech republic No. MSM0021622402.

\section{REFERENCES}

1. American Heart Association. Heart Disease and Stroke Statistics-2003 update. Dallas, TX: American Heart Association 2002.

2. Cavusoglu E, Chopra V, Gupta A, Ruwende C, Yanamadala S, Eng C, Clark LT, Pinsky DJ, Marmur JD. Usefullness of the white blood cell count as a predictor of angiographic findings in an unselected population referred for coronary angiography. Am J Cardiol 2006; 98:1189-1193.

3. Taniguchi H, Momiyama Y, Ohmori R, Yonemura A, Yamashita T, Tamai S, Nakamura H, Ohsuzu F. Associations of plasma C - reactive protein levels with the presence and extent of coronary stenosis in patients with stable coronary artery disease. Atherosclerosis 2005; 178:173-177.

4. Erren M, Reinecke H, Junker R, Fobker M, Schulte H, Schurek JO, Kropf J, Kerber S, Breithardt G, Assman G, Cullen P. Systemic inflammatory parameters in patients with atherosclerosis of the coronary and peripheral arteries. Arterioscler Thromb Vasc Biol 1999; 19(10):2355-2363.

5. Sabatine MS, Morrow DA, Cannon CP, Murphy SA, Demopoulos LA, DiBattiste PM, McCabe CH, Braunwald E, Gibson CM. Relationship between baseline white blood cell count and degree of coronary artery disease and mortality in patients with acute coronary syndromes: a TACTICS-TIMI 18 (Treat Angina with Aggrastat and determine Cost of Therapy with an Invasive or Conservative strategy-Thrombolysis In Myocardial Infarction 18 trial) substudy. J Am Coll Cardiol 2002; 40:1761-1768.

6. Rasouli M, Kiasari AM, Bagheri B. Total and differential leukocytes counts, but not hsCRP, ESR, and five fractioned serum proteins have significant potency to predict stable coronary artery disease. Clin Chim Acta 2007; 377:127-132.

7. Zebrack JS, Muhlestein JB, Horne BD, Anderson JL. C-reactive protein and angiographic coronary artery disease: Independent and additive predictors of risk in subject with angina. J Am Coll Cardiol 2002;39:632-7.

8. Madjid M, Awan I, Willerson JT, Casscells SW. Leukocyte count and coronary heart disease. J Am Coll Cardiol 2004; 44:1945-56.

9. Libby P. Molecular bases of the acute coronary syndromes. Circulation 1995; 91:2844-50.

10. Foussas SG, Zairis MN, Anastassios GL, Patsourakos NG, Tsirimpis VG, Katsaros K, Beldekos DJ, Handanis SM, Mytas DZ, Karidis KS, Tselioti PG, Prekates AA, Ambrose JA. Early prognostic usefullness of C-reactivwe protein added to the thrombolysis in myocardial infarction risk score in acute coronary syndromes. Am J Cardiol 2005; 96:533-537.

11. Cannon CP, McCabe CH, Wilcox RG, Bentley JH, Braunwald E for the OPUS-TIMI 16 investigators. Association of white blood cell count with increased mortality in acute myocardial infarction and unstable angina pectoris. Am J Cardiol 2001; 97:636-639.

12. Barron HV, Cannon CP, Murphy SA, Braunwald E, Gibson CM. Association between white blood cell count, epicardial blood flow, myocardial perfusion, and clinical outcomes in the setting of acute myocardial infarction: a Thrombolysis in Myocardial Infarction 10 substudy. Circulation 2000; 102:2329-2334.

13. Danesh J, Wheeler JG, Hirschfeld GM, Eda S, Eiriksdottir G, Rumley A, Lowe GDO, Pepys MB, Gudnason V. C-reactive protein 
and other circulating markers of inflammation in the prediction of coronary heart disease. N Engl J Med 2004; 350:1387-1397.

14. Brown DW, Giles WH, Croft JB. White blood cell count: an independent predictor of coronary heart disease mortality among a national cohort. J Clin Epidemiol 2001; 54:316-322.

15. Wheeler JG, Mussolino ME, Gillum RF, Danesh J. Associations between differential leukocyte count and incident coronary heart disease: 1764 incident cases from seven prospective studies of 30,374 individuals. Eur Heart J 2004; 25:1287-1292.

16. Danesh J, Collins R, Appleby P, Peto R. Association of fibrinogen, C-reactive protein, albumin, or leukocyte count with coronary heart disease. JAMA 1998; 279:1477-1482.

17. Liu MY, Hu DY. The predictive value of serum advanced fibrinogen and uric acid for acute coronary event risk. Zhongzhua Yi Xue Za Zhi 2006; 86(10):678-80.

18. Jelic-Ivanovic Z, Memon L, Spasojevic-Kalimanovska V, BogavacStanojevic N, Spasic S. Independent association of high serum uric acid concentration with angiographically defined coronary artery disease. Tohoku J Exp Med 2007; 211(4):369-77.

19. Bickel C, Ruprecht HJ, Blankenberg S, Rippin G, Hafner G, Daunhauer A, Hofmann KP, Meyer J. Serum uric acid as an inde- pendent predictor of mortality in patients with angiographically proven coronary artery disease. Am J Cardiol 2002; 89(1):12-7.

20. Levenson J, Giral P, Megnien JL, Gariepy J, Plainfosse MC, Simon A. Fibrinogen and its relations to subclinical extracoronary and coronary atherosclerosis in hypercholesterolemic men. Arterioscler Thromb Vasc Biol 1997; 17(1):45-50.

21. Levenson J, Giral P, Razavian M, Gariepy J, Simon A. Fibrinogen and silent atherosclerosis in subject with cardiovascular risk factors. Arterioscler Thromb Vasc Biol 1995; 15(9):1263-1268.

22. Bartnicky P, Stolarek R, Rysz J. Coronary artery atherosclerosis in patients with the initial and the early stage of chronic renal failure. Centr Eur J Med 2009; 4(1):32-36.

23. Cerne D, Kaplan-Pavlovic S, Kranjec I, Jurgens G. Mildly elevated serum creatinine concentration correlates with the extent of coronary atherosclerosis. Renal Failure 2000; 22(6):799-808.

24. Chade AR, Brosh D, Higano ST, Lennon RJ, Lerman LO, Lerman A. Mild renal insufficiency is associated with reduced coronary flow in patients with non-obstructive coronary artery disease. Kidney Int 2006; 69(2):266-271. 
